\title{
Application du modèle de Pitzer à l'étude de l'évaporation solaire isotherme des saumures naturelles
}

\author{
F. Khlissa, A. M'nif et R. Rokbani \\ Laboratoire des Procédés Chimiques, Institut National de Recherche Scientifique \\ et Technique, BP. 95, 2050 Hammam-Lif, Tunisie
}

\begin{abstract}
The brine reserves of southern Tunisian chotts and sebkhats represent a source of mineral salts of a great interest, from which mineral salts can be recovered by isothermal solar evaporation. The evolution of density and ionic composition of the treated brine, during its isothermal evaporation, was studied. The experimental pathway of the evaporated brine was plotted on the oceanic quinary diagram $\mathrm{Na}^{+}, \mathrm{K}^{+}, \mathrm{Mg}^{2+}, \mathrm{Cl}^{-}, \mathrm{SO}_{4}{ }^{2-} / / \mathrm{H}_{2} \mathrm{O}$. In order to better understanding the observed chemical equilibria and, especially, to predict the precipitation sequences of mineral salts, during solar evaporation at $25^{\circ} \mathrm{C}$, the theoretical pathway of the same studied brine was calculated using Pitzer's ion interaction model. The experimental, the theoretical and the calculated pathways are not the same.
\end{abstract}

\section{INTRODUCTION}

La saumure naturelle de Sebkhat El Melah de Zarzis (Sud-Est Tunisien) est une saumure fortement magnésienne dont la teneur en magnésium est de l'ordre 54 g.L $\mathrm{L}^{-1}$. L'évaporation de cette saumure est confrontée à plusieurs difficultés, dues à l'abondance du cation $\mathrm{Mg}^{2+}$ [1]. En effet, ce dernier forme avec l'eau le complexe $\left[\mathrm{Mg}\left(\mathrm{H}_{2} \mathrm{O}\right)_{6}\right]^{2+}$, entraînant ainsi la rétention d'une quantité importante d'eau et l'arrêt de l'évaporation après la réduction de $50 \%$ du volume initial de la saumure. Dans le but de résoudre ce problème et d'augmenter le rendement de l'opération d'extraction des sels minéraux par évaporation, des traitements chimiques spécifiques, visant la réactivation de ce type de saumure, en diminuant sa teneur en magnésium, ont été employés [2].

L'étude expérimentale et théorique, à l'aide du modèle de Pitzer [3], de l'évaporation isotherme à $25^{\circ} \mathrm{C}$ de la saumure de sebkhat El Melah de Zarzis, traitée fait l'objet du présent travail. Aussi, les cheminements expérimental et théorique des points représentatifs de cette saumure au cours de son évaporation ont été tracés sur le diagramme quinaire océanique $\mathrm{Na}^{+}, \mathrm{K}^{+}, \mathrm{Mg}^{2+}, \mathrm{Cl}^{-}, \mathrm{SO}_{4}^{2-} / / \mathrm{H}_{2} \mathrm{O}$, à $25^{\circ} \mathrm{C}$.

\section{MÉTHODOLOGIE DE L'ÉTUDE}

Le traitement chimique de la saumure naturelle de sebkhat El Melah de Zarzis a permis de réduire sa teneur en magnésium de $88.2 \%$. La saumure traitée ainsi obtenue a fait l'objet de la présente étude. En effet, l'évaporation isotherme de cette saumure traitée a été étudiée tout d'abord expérimentalement et ensuite théoriquement en faisant appel au programme « SolubLTS » [4] qui est un programme de calcul sur Mathcad, se basant sur le modèle d'interaction ionique de Pitzer. 


\subsection{Mode opératoire}

Deux litres de la saumure traitée dont la composition est donnée dans le tableau 1 ont été évaporés dans un cristallisoir. Ce dernier est placé dans un bain marie dont la température est maintenue constante, à $25^{\circ} \mathrm{C}$, à l'aide d'une tête chauffante. L'évolution de la densité et de la composition ionique de la phase liquide ainsi que la nature des sels déposés, au cours de l'évaporation, sont suivies systématiquement. En effet, après la séparation de phases formées, par filtration, les phases solides sont caractérisées par analyses chimiques et diffraction des RX, alors que les phases liquides sont caractérisées par analyses chimiques et par mesure de la densité.

Le cheminement expérimental représentant l'évolution de la composition ionique de la saumure traitée au cours de son évaporation est ensuite tracé sur le diagramme quinaire océanique $\mathrm{Na}^{+}, \mathrm{K}^{+}$, $\mathrm{Mg}^{2+}, \mathrm{Cl}^{-}, \mathrm{SO}_{4}^{2-} / / \mathrm{H}_{2} \mathrm{O}$, à $25^{\circ} \mathrm{C}$.

Tableau 1. Molalités des différents ions dans les saumures étudiées.

\begin{tabular}{|l|l|l|l|l|l|l|}
\hline & $\mathrm{Na}^{+}$ & $\mathrm{K}^{+}$ & $\mathrm{Mg}^{2+}$ & $\mathrm{Cl}^{-}$ & $\mathrm{SO}_{4}^{2-}$ & $\begin{array}{l}\% \text { molaire } \\
\text { d'élimination de Mg. }\end{array}$ \\
\hline S. N. El Meleh & 1.998 & 0.207 & 2.450 & 6.316 & 0.344 & 0 \\
\hline S. traitée & 4.345 & 0.147 & 0.289 & 4.489 & 0.356 & 88.20 \\
\hline
\end{tabular}

\subsection{Calcul de la solubilité}

Le modèle d'interaction ionique de Pitzer a été utilisé dans ce travail pour calculer le cheminement théorique de la saumure traitée au cours de son évaporation isotherme à $25^{\circ} \mathrm{C}$. En effet, à l'aide du programme SolubLTS qui est un programme de calcul sur Mathcad, il a été possible de déterminer les molalités du magnésium dissous, des chlorures dissous et celles de l'astrakanite $\left(\mathrm{Na}_{2} \mathrm{Mg}\left(\mathrm{SO}_{4}\right)_{2} \cdot 4 \mathrm{H}_{2} \mathrm{O}\right)$ en fonction des molalités du potassium et du sodium, déterminées expérimentalement, par analyses chimiques.

Les molalités recherchées des espèces ioniques dans les solutions saturées sont déterminées en considérant les équilibres suivants :

- Produit de solubilité de la halite $(\mathrm{NaCl})$ :

$$
\mathrm{K}_{\mathrm{S} \text { halite }}=\gamma_{\mathrm{Na}^{+}} \mathrm{m}_{\mathrm{Na}^{+}} \gamma_{\mathrm{Cl}} \mathrm{m}_{\mathrm{Cl}}
$$

- $\quad$ Produit de solubilité de l'astrakanite :

$$
\mathrm{K}_{\mathrm{S}_{\text {astrakanit }}}=\gamma_{\mathrm{Na}^{+}}^{2} \mathrm{~m}_{\mathrm{Na}^{+}}^{2} \gamma_{\mathrm{Mg}^{2+}} \mathrm{m}_{\mathrm{Mg}^{2+}} \gamma_{\mathrm{SO}_{4}^{2-}}^{2} \mathrm{~m}_{\mathrm{SO}_{4}^{2-}}^{2} \mathrm{a}_{\mathrm{H}_{2} \mathrm{O}}^{4}
$$

\section{RÉSULTATS ET DISCUSSION}

Les évolutions de la densité et des compositions expérimentales et calculées de la saumure traitée (exprimées en molalités : moles par kilogrammes du solvant), au cours de son évaporation isotherme à $25^{\circ} \mathrm{C}$, sont données dans le tableau 2. 
Tableau 2. Evolution de la composition ionique (exprimées en molalités) et de la densité.

\begin{tabular}{|l|c|c|c|c|c|c|c|c|c|c|}
\hline & $\mathbf{d}$ & $\mathbf{m}_{\mathrm{Na}^{+}}$ & $\mathbf{m}_{\mathbf{K}^{+}}$ & $\mathbf{m}_{\mathbf{M g}^{2+}}$ & $\mathbf{m}_{\mathbf{C l}^{-}}$ & $\mathbf{m}_{\mathbf{S O}_{4}^{2-}}$ & Phases solides & $\mathbf{m}_{\mathbf{M g d i s}}$ & $\mathbf{m}_{\mathbf{C l d i s}}$ & $\mathbf{m}_{\text {astrakanite }}$ \\
\hline $\mathbf{A}$ & 1.109 & 4.345 & 0.147 & 0.289 & 4.489 & 0.356 & $* *$ & 0.416 & 0.831 & 0.540 \\
\hline $\mathbf{A}_{\mathbf{1}}$ & 1.224 & 4.439 & 0.180 & 0.367 & 4.534 & 0.440 & halite + epsomite & 0.342 & 0.684 & 0.557 \\
\hline $\mathbf{A}_{\mathbf{2}}$ & 1.229 & 4.391 & 0.191 & 0.474 & 4.591 & 0.541 & halite + epsomite & 0.368 & 0.736 & 0.553 \\
\hline $\mathbf{A}_{\mathbf{3}}$ & 1.233 & 4.666 & 0.201 & 0.612 & 4.801 & 0.650 & halite + epsomite & 0.183 & 0.365 & 0.596 \\
\hline $\mathbf{A}_{\mathbf{4}}$ & 1.253 & 4.654 & 0.205 & 0.901 & 5.308 & 0.832 & halite + epsomite & 0.189 & 0.378 & 0.595 \\
\hline $\mathbf{A}_{\mathbf{5}}$ & 1.271 & 4.720 & 0.213 & 1.188 & 5.341 & 0.918 & halite + epsomite & 0.140 & 0.280 & 0.609 \\
\hline $\mathbf{A}_{\mathbf{6}}$ & 1.293 & 4.792 & 0.225 & 1.264 & 5.325 & 0.908 & halite + epsomite & 0.084 & 0.169 & 0.626 \\
\hline
\end{tabular}

Le cheminement expérimental, le cheminement théorique prévisible ainsi que le cheminement calculé à l'aide du modèle de Pitzer sont représentés sur la projection de Jänecke du diagramme quinaire océanique $\mathrm{Na}^{+}, \mathrm{K}^{+}, \mathrm{Mg}^{2+}, \mathrm{Cl}^{-}, \mathrm{SO}_{4}{ }^{2-} / / \mathrm{H}_{2} \mathrm{O}$, à $25^{\circ} \mathrm{C}$, donné à la figure 1 . Il est à noter que ce système a été étudié par Van't Hoff à différentes températures entre $25^{\circ} \mathrm{C}$ et $83^{\circ} \mathrm{C}$. Différentes représentations ont été proposées pour ce système. Nous avons adopté celle de Jänacke qui correspond à une projection centrale, sur un triangle équilatéral, du volume enveloppant les solutions saturées en halite $(\mathrm{NaCl})$. De ce fait, un domaine de précipitation d'un sel sur cette projection correspond, en réalité à l'apparition de deux phases solides dont l'une est obligatoirement formée de la halite.

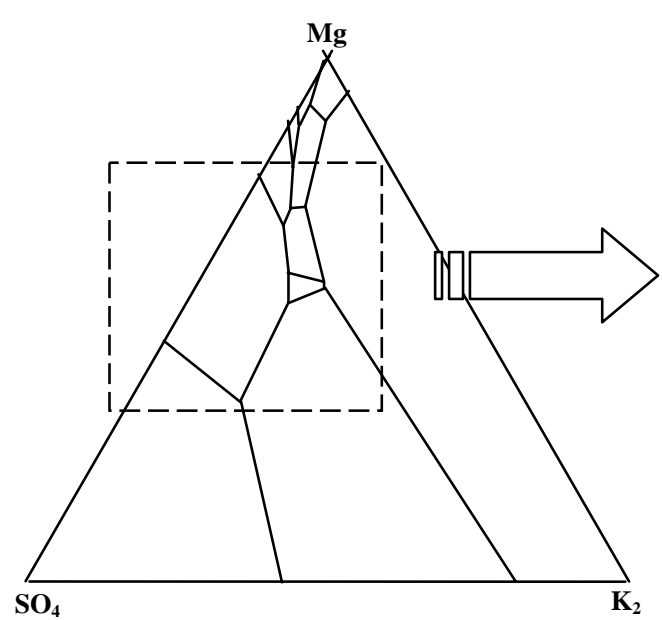

- Cheminement expérimental

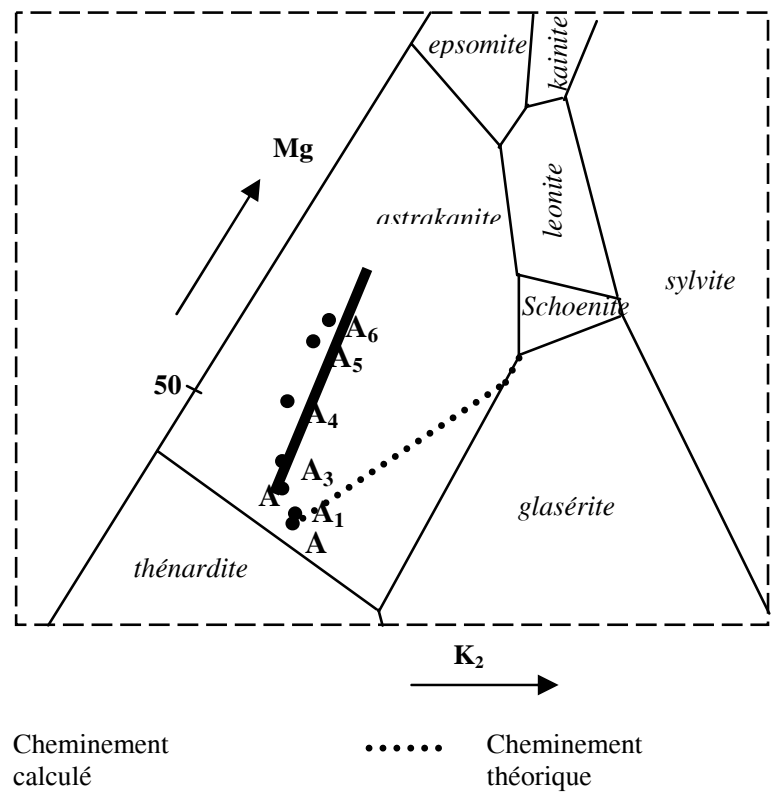

calculé théorique

Figure 1. Diagramme quinaire océanique et cheminements expérimental, théorique et calculés à $25^{\circ} \mathrm{C}$. 
Les lieux des points mélanges, représentés sur le diagramme de la figure 1, ont été déterminés en appliquant les équations des coordonnées de Jänecké suivantes :

$$
\begin{aligned}
& \% \mathrm{Mg}=\frac{100 \times \mathrm{n}_{\mathrm{Mg}}}{\mathrm{D}} \\
& \% \mathrm{~K}_{2}=\frac{100 \times \frac{\mathrm{n}_{\mathrm{K}}}{2}}{\mathrm{D}} \\
& \% \mathrm{SO}_{4}=\frac{100 \times \mathrm{n}_{\mathrm{SO}_{4}}}{\mathrm{D}}
\end{aligned}
$$

Avec $\quad \mathrm{D}=\mathrm{n}_{\mathrm{Mg}}+\frac{\mathrm{n}_{\mathrm{K}}}{2}+\mathrm{n}_{\mathrm{SO}_{4}}$ et $\mathrm{n}_{\mathrm{Mg}}, \mathrm{n}_{\mathrm{K}}, \mathrm{n}_{\mathrm{SO}_{4}}$ sont les nombres des moles, respectivement, du magnésium, du potassium et des sulfates.

Le cheminement théorique prévoit la précipitation, en premier lieu, d'un mélange formé de 2 sels qui sont la halite $(\mathrm{NaCl})$ et l'astrakanite. Cette première séquence de cristallisation sera suivie par une seconde séquence correspondant à la précipitation de la halite $(\mathrm{NaCl})$, de l'astrakanite et de la glasérite $\left(\mathrm{NaK}_{3}\left(\mathrm{SO}_{4}\right)_{2}\right)$. Contrairement à ces prévisions théoriques, l'étude expérimentale de cette évaporation a mis en évidence une seule séquence cristalline pendant laquelle l'epsomite $\left(\mathrm{MgSO}_{4} \cdot 7 \mathrm{H}_{2} \mathrm{O}\right)$ coprécipite avec la halite, à partir d'une densité de 1.224. Cette anomalie est expliquée par des considérations structurales et cinétiques de la formation de ces sels. En effet, il est impossible d'obtenir l'astrakanite par simple évaporation et il faut attendre au moins 9 jours pour former ce sel à partir d'un mélange halite-epsomite [5].

L'écart enregistré entre les cheminements expérimental et calculé est dû à la nature des données expérimentales $\left(\mathrm{m}_{\mathrm{Na}}\right.$ et $\left.\mathrm{m}_{\mathrm{K}}\right)$ utilisées dans ces calculs. En effet, ces données relatives à des solutions sursaturées et des équilibres métastables, tandis que les constantes du modèle et les équations (produits de solubilité de la halite et de l'astrakanite employées dans nos calculs sont relatives à des équilibres stables.

\section{Références}

[1] C. Djebali, L. Zayani, A. M'Nif et R. Rokbani, Journal de la société chimique de Tunisie, $\mathbf{4}, n^{\circ} 3$ (1998) 233-243

[2] F. Khlissa, A. M'nif et R. Rokbani, Russian Journal of Inorganic Chemistry, 49, n 4 (2004) 653-659 (from Zhurnal Neorganicheskoi Khimii, 49, $\mathrm{n}^{\circ} 4$ (2004) 715-720

[3] K. S. Pitzer, Activity Coefficients in Electrolyte Solutions, 2nd Edition (CRC Presse Ince., Boca Raton, Florida) 1991

[4] F. Marliacy, Thèse de Doctorat, Inst. Nat. Polytech. de Lorraine, Ecole Nationale Supérieure des Industries Chimiques, juin 1999

[5] A. M’Nif et R. Rokbani, Crystal Research and Technology, 39, n 1 (2004) $40-49$ 